\title{
(-)-Oleocanthal exerts anti-melanoma activities and inhibits STAT3 signaling pathway
}

\author{
YANLI GU, JING WANG and LIXIN PENG \\ Department of Dermatology, Daqing Oilfield General Hospital, Saertu, Daqing, Heilongjiang 163001, P.R. China
}

Received June 15, 2016; Accepted November 1, 2016

DOI: $10.3892 /$ or.2016.5270

\begin{abstract}
Tumor angiogenesis, growth and metastasis are three closely related processes. We therefore explored the effects of (-)-oleocanthal (OC) on the three processes in melanoma and investigated underlying mechanisms. In vitro, OC suppressed proliferation, migration, invasion and induced apoptosis in melanoma cells. In addition, OC inhibited proliferation, migration, invasion and tube formation in human umbilical vascular endothelial cells. In vivo, it exhibited potent activity in suppressing tumor growth in a subcutaneous xenograft model. Furthermore, OC suppressed proliferation and angiogenesis as measured by immunohistochemical staining of $\mathrm{Ki}-67$ and $\mathrm{CD} 31$. In addition, OC was found to inhibit metastasis of melanoma in a lung metastasis model. Mechanistically, OC significantly suppressed signal transducer and activator of transcription 3 (STAT3) phosphorylation, decreased STAT3 nuclear localization and inhibited STAT3 transcriptional activity. OC also downregulated STAT3 target genes, including Mcl-1, Bcl-xL, MMP-2, MMP-9, VEGF, which are involved in apoptosis, invasion and angiogenesis of melanoma. These results support further investigation of OC as a potential anti-melanoma drug.
\end{abstract}

\section{Introduction}

Melanoma, a malignant tumor of skin cancer, is often identified as one of the most deadly of tumors. The incidence of melanoma has continued to increase in the past decades (1). Despite the variety of therapies used to treat melanoma, the prognosis for melanoma remains very poor, with a 5-year

Correspondence to: Dr Yanli Gu, Department of Dermatology, Daqing Oilfield General Hospital, 9 ZhongKang Street, Daqing, Heilongjiang 163001, P.R. China

E-mail: dyydam@126.com

Abbreviations: OC, (-)-oleocanthal; VEGF, vascular endothelial growth factor; MMP-2, matrix metalloproteinase-2; MMP-9, matrix metalloproteinase-9; HCC, hepatocellular carcinoma; STAT3, signal transducer and activator of transcription 3

Key words: melanoma, (-)-oleocanthal, growth, metastasis, signal transducer and activator of transcription 3 survival rate of $<5 \%(2,3)$. Most therapeutics such as BRAF inhibitors, cytotoxic $\mathrm{T}$ lymphocyte-associated antigen 4 antibody and interleukin-2 biological therapy have failed in melanoma treatment because of the low response rates and significant toxicities (4-6). Therefore, new candidates for melanoma that possess potential antitumor activity and low toxicity are urgently needed.

Many signaling pathways are involved in the progression of melanoma, including signal transducer and activator of transcription 3 (STAT3) signaling pathway (7). STAT3 is constitutively activated in $50-90 \%$ of melanoma and the high expression of phosphorylated STAT3 (p-STAT3) is associated with melanoma progression $(8,9)$. STAT3 activity increased the invasive ability of melanoma and is required for active melanogenesis by regulating tyrosinase gene expression and enzyme activity (10). The activation of STAT3 can stimulate brain metastasis of melanoma, and upregulate the expression of vascular endothelial growth factor (VEGF) and matrix metalloproteinase-2 (MMP-2) (9). Constitutive STAT3 activation promotes melanoma growth and angiogenesis by increasing the expression of VEGF (11). Moreover, cumulative evidence shows that blockade of STAT3 by small molecule inhibitors can suppress the growth, angiogenesis and metastasis of melanoma cells (12-14). Therefore, STAT3 may be a critical target for melanoma therapy.

Accumulating evidence demonstrates that natural compounds present in the human diet may significantly alter the natural history of a malignant tumor (15). Recently, there has been growing interest in the role of nutrition in melanoma chemoprevention (16). Olive oil is the main source of dietary fat in the Mediterranean diet, the consumers of which have a low incidence of cardiovascular disease, age related cognitive disease, and cancer $(17,18)$. (-)-Oleocanthal (OC), a main ingredient contained in virgin olive oil (VOO), has been reported to possess various biological activities such as anti-oxidative, anti-bacterial and anti-inflammatory activities (19-23). In addition, OC exhibits potent antitumor abilities in many malignant tumors, including breast carcinoma, prostate carcinoma and multiple myeloma (24-27). Moreover, OC has also been identified as a STAT3 inhibitor in human hepatocellular carcinoma (HCC) (28). However, the role of $\mathrm{OC}$ in melanoma remains unclear. In the present study, we investigated whether OC inhibited the growth and metastasis of melanoma through the blockage of STAT3 signaling pathway. 


\section{Materials and methods}

Cell lines, reagents and antibodies. A375, A2058, HUVEC and $\mathrm{HaCaT}$ cell lines were purchased from the Cell Resource Center of Shanghai Institutes for Biological Sciences, Chinese Academy of Sciences (Shanghai, China). All the cells were cultured in Dulbecco's modified Eagle's medium (DMEM; Gibco, Grand Island, NY, USA) supplemented with $10 \%$ fetal bovine serum (FBS). Z-VAD-FMK were purchased from Sigma-Aldrich (St. Louis, MO, USA). Primary antibodies against Bcl-xL, Mcl-1, cleaved-caspase-3, cleaved-caspase-9, cleaved-PARP, p-STAT3 and STAT3 were purchased from Cell Signaling Technology (Danvers, MA, USA). GAPDH and secondary antibodies against mouse IgG-horseradish peroxidase (HRP) and rabbit IgG-HRP were obtained from Santa Cruz Biotechnology, Inc. (Santa Cruz, CA, USA). Antibodies against Ki-67, CD31, VEGF, MMP-9 and MMP-2 were obtained from Abcam (Cambridge, MA, USA).

Extraction and isolation of $O C$. Approximately 2 liters of $\mathrm{n}$-hexane and $1 \mathrm{~kg}$ of EVOO (Sigma-Aldrich) were mixed, and then $\mathrm{CH}_{3} \mathrm{CN}-\mathrm{MeOH}$ (1 liter, 20:80) was added and shaken twice. Dried organic layer (24 g) was subjected to repeated medium pressure liquid chromatography (MPLC) in a $50 \times 3 \mathrm{~cm}$ column on lipophilic Sephadex LH20 (bead size 25-100 $\mu \mathrm{m}$; Sigma-Aldrich) using n-hexane- $\mathrm{CH}_{2} \mathrm{Cl}_{2}$ (1:9), isocratic elution, followed by MPLC (10 g, 25x $1 \mathrm{~cm}$ column) on C-18 reversed-phase silica gel with Bakerbond octadecyl ( $40 \mu \mathrm{m}$; Mallinckrodt Baker, Inc.) to afford $13.3 \mathrm{mg}$ of OC with $>97 \%$ purity (HPLC) along with several other impure fractions. Identification and purity of 1 were also based on comparison of its ${ }^{1} \mathrm{H}$ and ${ }^{13} \mathrm{C}$ NMR data with the literature (29). Generally, 1:100 ratios of mixtures to be chromatographed versus the stationary phase were used in all liquid chromatographic purifications.

Establishment of stable A375-luciferase cell line. Lentiviral vectors encoding the human Firefly Luciferase gene were constructed by GeneChem Corporation (Shanghai, China). The empty vector was used as a negative control. The lentiviral vectors were transfected into A375 cells with a multiplicity of infection (MOI) of 60 in the presence of polybrene $(5 \mu \mathrm{g} / \mathrm{ml})$. At $48 \mathrm{~h}$ after transfection, transfected cells were selected for 2 weeks with $2.5 \mu \mathrm{g} / \mathrm{ml}$ puromycin (Sigma-Aldrich). Cells, which were obtained 2 weeks after drug selection without subcloning, were used for further experiments.

Cell viability assay. Cell viability was determined using CCK-8 assay (Dojindo Laboratories, Kumamoto, Japan) according to the manufacturer's instruction. Melanoma, HaCaT or HUVEC cells $\left(3 \times 10^{3}\right)$ were seeded into a well of a 96-well plate and cultured in $150 \mu \mathrm{l}$ of medium supplemented with $10 \%$ FBS. After $24 \mathrm{~h}, \mathrm{OC}(0-60 \mu \mathrm{M})$ was added into the culture medium. Then, after the cells were incubated at $37^{\circ} \mathrm{C}$ for different times ( 24 or $48 \mathrm{~h}$ ), the medium was changed to $100 \mu \mathrm{l}$ of medium and $10 \mu \mathrm{l}$ of CCK- 8 reagent. The cells were incubated for $2 \mathrm{~h}$ at $37^{\circ} \mathrm{C}$. Finally, the optical density was measured using an EnSpire $^{\text {TM }} 2300$ Multilabel Reader (PerkinElmer, Waltham, MA, USA) at $450 \mathrm{~nm}$.
Colony formation assay. Ten thousand A375 cells were cultured in 6-well plates for 12 days. Fresh medium with OC was added to the plates every 3 days. At the end of treatment, colonies were stained with $0.05 \%$ crystal violet for $30 \mathrm{~min}$ and counted.

Cell cycle assay. The cell cycle analysis kit was purchased from BD Biosciences (San Jose, CA, USA). Melanoma cells were seeded into 6 -well plates at $4 \times 10^{5} / \mathrm{dish}$. After incubation with OC ( 20 or $40 \mu \mathrm{M})$ for $48 \mathrm{~h}$, cells were trypsinized and washed twice with PBS. Then cells were cultured with Reagents A-C according to the manufacturer's protocol and subjected to flow cytometry.

Apoptosis assay. The Annexin V-FITC apoptosis kit was purchased from BD Biosciences. Melanoma cells $\left(4 \times 10^{5}\right.$ cells/well) were incubated with OC $(20$ or $40 \mu \mathrm{M})$ for $48 \mathrm{~h}, 1 \times 10^{4}$ cells were collected and washed twice with cold PBS. Then apoptotic cells were evaluated by double staining with propidium iodide (PI) and Annexin V labeled with FITC according to the manufacturer's instruction.

Wound-healing assay. Melanoma cells were seeded at a plating density of $4 \times 10^{5} /$ well in 6-well plates and cultured overnight. After reaching $100 \%$ confluence, the cells were gently scraped with aplastic tip to produce a wound area. After wounding cells were incubated with fresh DMEM medium with $10 \mu \mathrm{M}$ of OC, and the cell movement throughout the wound area was examined after $24 \mathrm{~h}$.

Migration and invasion assays. Migration assay was performed using BioCoat Chambers (BD Biosciences). DMEM containing $10 \%$ FBS was placed in the lower chamber as a chemoattractant. Cells $\left(4 \times 10^{5}\right)$ were seeded to the upper chamber in $200 \mu 1$ of serum-free medium containing different concentrations of $\mathrm{OC}$, and allowed to migrate for $24 \mathrm{~h}$. Then, the cells on the upper surface of the membrane were removed. Chambers were fixed in $4 \%$ paraformaldehyde for $5 \mathrm{~min}$ and stained with crystal violet. The cells that penetrated the filter were observed with a microscope and cells from 5 random fields were counted. Invasion assay was carried out under the same conditions as the migration assay, except that the chambers were coated with Matrigel.

Capillary-like tube formation assay. Tube formation was assessed as described previously (30). Briefly, HUVECs were pretreated with various dilutions of $\mathrm{OC}$ for $24 \mathrm{~h}$ and then seeded onto the Matrigel layer in 96-well plates at a density of $1 \times 10^{4}$ cells. After $8-12 \mathrm{~h}$, tubular structure of endothelial cells was photographed using an inverted microscope.

Luciferase reporter assay. Luciferase reporter assay was performed as described previously (30). Luciferase activity was determined with the Promega luciferase assay kit.

Western blotting. Melanoma cells were plated at a density of $4 \times 10^{5} /$ well on 6 -well plates. After incubation with different concentrations of $\mathrm{OC}$, the cells were washed twice with icecold PBS and treated with $120 \mu 1$ sample buffer on ice for $30 \mathrm{~min}$. The cell lysate was centrifuged at $12,000 \mathrm{rpm}$ for 
A
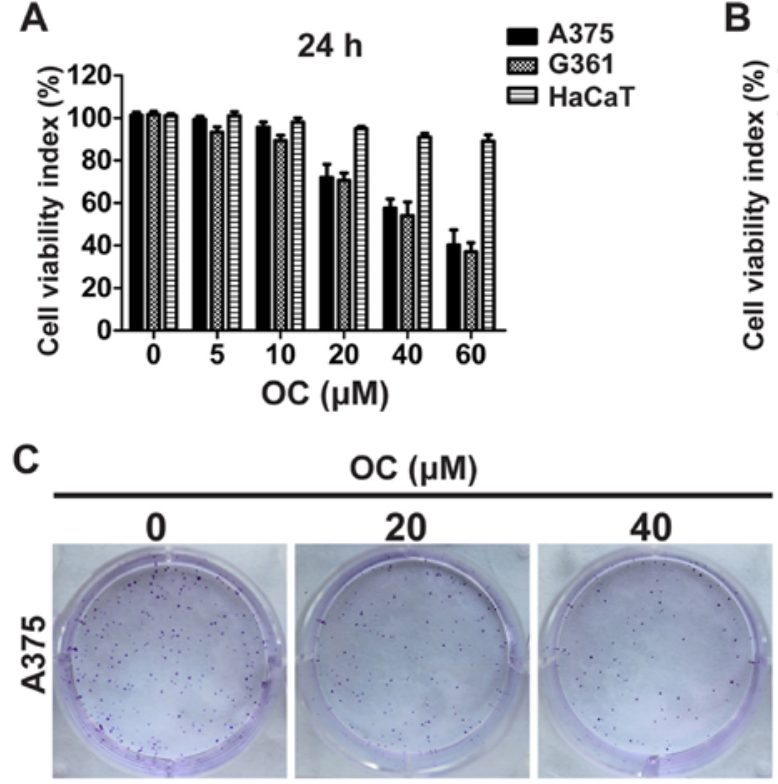

B
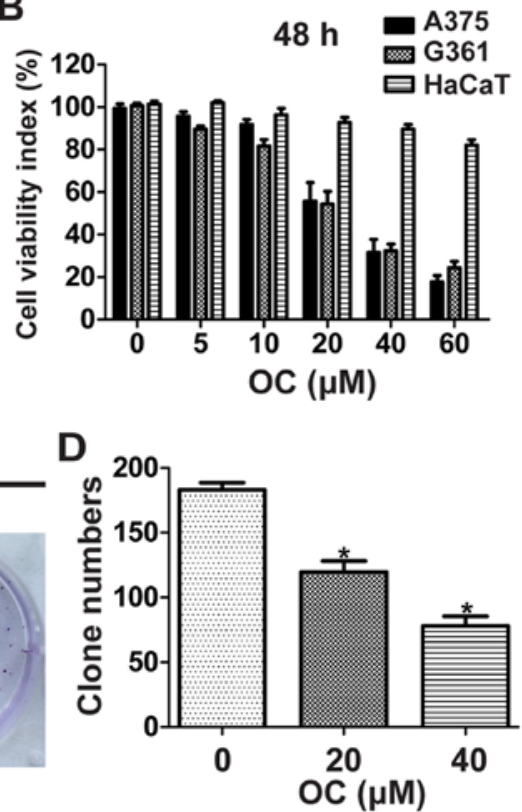

Figure 1 . OC suppresses the viability and colony formation of melanoma cells. (A) Melanoma and HaCaT cells were treated with $0,5,10,20,40$ or $60 \mu \mathrm{M}$ of OC for $24 \mathrm{~h}$, and cell viability was measured using the CCK-8 assay. (B) Melanoma and HaCaT cells were treated with 0, 5, 10, 20, 40 or 60 $\mu \mathrm{M}$ of OC for $48 \mathrm{~h}$, and cell viability was measured using the CCK-8 assay. (C) Representative images of foci formation assay of A375 cells are shown. (D) The number of foci was counted. Data are presented as the means \pm SD of three independent experiments. ${ }^{*} \mathrm{P}<0.05$.

$10 \mathrm{~min}$ at $4^{\circ} \mathrm{C}$. Protein lysates $(30 \mu \mathrm{l})$ were electrophoresed on a 12 or $10 \%$ SDS gel. Then the proteins were electrotransferred to a PVDF membrane and the membrane was blocked for 30 min with blocking solution (5\% non-fat dry milk in PBS-0.5\% Tween-20). The membrane was then incubated overnight at $4^{\circ} \mathrm{C}$ with primary antibodies (1:1,000). After that the membrane was washed in PBST for $30 \mathrm{~min}$, exposed to HRP-conjugated secondary antibody (diluted 1:2,000), and washed again in PBST for $30 \mathrm{~min}$. Final detection was performed using enhanced chemi-luminescence solution for $5 \mathrm{~min}$.

Histopathological analysis. The tumor tissues were isolated to perform hematoxylin and eosin staining and immunohistochemical staining. The tumor tissues were immediately fixed with $4 \%$ paraformaldehyde, sectioned, and stained using hematoxylin and eosin for light microscopy. For immunohistochemical staining, the non-specific binding was blocked with $1 \%(\mathrm{w} / \mathrm{v})$ bovine serum albumin at room temperature for $30 \mathrm{~min}$. The sections were then incubated with anti-Ki-67 and anti-CD31 antibodies. The slides were incubated with biotinylated secondary antibody for $1 \mathrm{~h}$ at room temperature. Finally, the sections were developed with DAB color solution (50 $\mu \mathrm{l} / \mathrm{section})$ for $5 \mathrm{~min}$ at room temperature. The number of positive cells were mounted and examined under a light microscope.

Animal experiments. Male BALB/c athymic nude mice (5 weeks old) were obtained from the experimental animal center of Shanghai Institute for Biological Sciences (Shanghai, China) and housed under standard conditions and cared for according to the institutional guidelines for animal care. All procedures and care administered to the animals were approved by the Institutional Ethics Committee. We used six mice in each group to establish subcutaneous xenograft model. A 375 cells $\left(4 \times 10^{6}\right)$ in $200 \mu 1$ of PBS were subcutaneously injected into the flanks of nude mice. After the tumor volume reached $100 \mathrm{~mm}^{3}$, OC (10 mg/kg/day) was administered i.p. for 3 weeks. At the end of treatment, tumors were excised and tumor volume was calculated by using the following equation: Tumor volume $=$ length $\mathrm{x}(\text { width })^{2} \mathrm{x} \pi / 6$

For the lung metastasis model, A375 cells $\left(5 \times 10^{6}\right)$ in $200 \mu \mathrm{l}$ of PBS were injected into the nude mice through the tail vein ( $n=6 /$ group). Animals were randomized to receive either OC (15 mg/kg/day) or DMSO at 1 week after injection. Tumor metastasis was imaged and quantified by bioluminescence every two weeks. After 6 weeks, the mice were sacrificed, and their lungs were collected for further experiment.

Statistical analysis. Data are presented as mean values \pm standard deviation (SD). Comparisons between multiple groups were performed using the one-way analysis of variance (ANOVA) followed by Dunnett's t-test. A value of $\mathrm{P}<0.05$ was considered statistically significant.

\section{Results}

OC suppresses proliferation and colony formation of melanoma cells. CCK-8 assay was first performed to detect the anti-proliferative effect of OC on melanoma cells. Melanoma cells (A375 and A2058 cells) were cultured in different concentrations $(0-60 \mu \mathrm{M})$ for $24 \mathrm{~h}$, and then cell viability was measured. OC significantly inhibited proliferation of melanoma cells in a dose-dependent manner (Fig. 1A). Extending drug exposure to $48 \mathrm{~h}$ resulted in additional cytotoxicity, indicating that OC also suppressed proliferation of melanoma cells in a time-dependent manner (Fig. 1B). In addition, OC treatment showed lesser toxicity on HaCaT cells (a keratinocyte cell line 
A
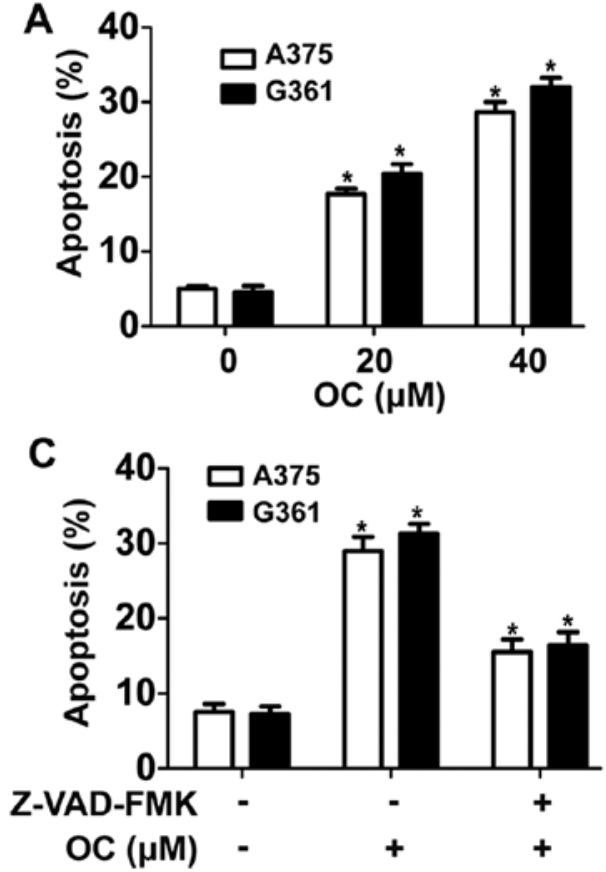

B

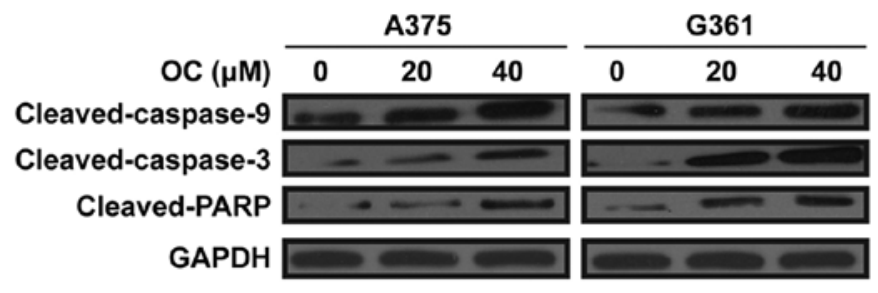

D

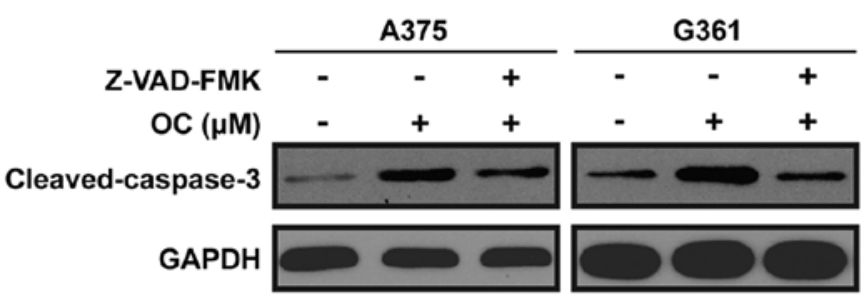

Figure 2. OC induces cell apoptosis in a caspase-dependent manner. (A) Flow cytometry results of Annexin V-PI-stained melanoma cells after exposure to 0, 20 or $40 \mu \mathrm{M}$ of OC for $48 \mathrm{~h}$. (B) Lysates from melanoma cells treated with 0,20 or $40 \mu \mathrm{M}$ of OC for $48 \mathrm{~h}$ were probed for cleaved-caspase-9, cleaved-caspase- 3 and cleaved-PARP by western blotting. (C) Changes in histograms of the apoptotic rate from melanoma cells treated with DMSO, OC and OC in combination with the pan-caspase inhibitor, (Z-VAD-FMK). (D) Changes in the expression of cleaved-caspase-3 from melanoma cells treated with DMSO, OC and OC in combination with the pan-caspase inhibitor, (Z-VAD-FMK). Data are presented as the means \pm SD of three independent experiments. ${ }^{*}<0.05$.

from adult human skin), which suggested that $\mathrm{OC}$ was more potent to cancer cells than normal cells (Fig. 1A and B). Colony formation ability was then investigated to determine the longterm impact of OC on melanoma cell growth. As is shown in Fig. 1C and D, the colony formation ability of A375 cells was significantly inhibited by $\mathrm{OC}$ in a dose-dependent manner.

OC induces cell apoptosis in a caspase-dependent manner. To determine whether anti-proliferation effect induced by $\mathrm{OC}$ involved apoptosis, flow cytometric analysis with Annexin V-PI staining was performed. OC induced apoptosis of A375 and A2058 cells in a dose-dependent manner after OC treatment for $48 \mathrm{~h}$ (Fig. 2A). To investigate the signaling cascade which mediated OC-induced apoptosis, key proteins of capase pathway were detected. Results showed that OC significantly increased the expression of cleaved-caspase-9, cleaved-caspase-3 and cleaved-PARP in a dose-dependent manner (Fig. 2B). To explore the dependence of OC-induced apoptosis on the caspase pathway, melanoma cells were pretreated with a pan-caspase inhibitor, Z-VAD-FMK (10 mmol/l), before treatment with OC. The pretreatment with Z-VAD-FMK partially reduced OC-induced apoptosis as determined by Annexin V-PI staining (Fig. 2C). Western blotting was performed to determin whether Z-VAD-FMK suppressed OC-induced activation of caspase-3. Data showed that $\mathrm{OC}$-induced activation of caspase-3 was partially reversed by pretreatment of Z-VAD-FMK (Fig. 2D). Then, flow cytometric analysis was performed to evaluate the effect of $\mathrm{OC}$ on cell cycle progression. However, there was no apparent change in the distribution of the cell cycle after OC treatment (data not shown). These data showed that OC induce apoptosis in melanoma cells, partially in a caspase-dependent manner.
OC inhibits migration and invasion of melanoma cells. To investigate the effect of $\mathrm{OC}$ on melanoma cell migration, wound-healing assay was performed by using $10 \mu \mathrm{M}$ of $\mathrm{OC}$ to treat melanoma cells. OC at such doses did not affect cell proliferation, so its anticancer metastasis effect can be further studied unambiguously at noncytotoxic doses. Data showed that $\mathrm{OC}$ treatment significantly decreased the migration of A375 and A2058 cells (Fig. 3A and B). Matrigel-coated Transwell was then used to explore the effect of OC on the invasion ability of melanoma cells. Data showed that the invasive ability of A375 and A2058 cells was significantly suppressed after OC treatment (Fig. 3C and D).

OC suppresses proliferation, migration, invasion and tube formation of human umbilical vascular endothelial cells (HUVECs). Angiogenesis plays a critical role in the growth and metastasis of malignant tumors as the newly formed tumor vasculature serve initially as feeding tubes providing nutrients and oxygen for the tumor mass (31). Therefore, we next investigated the anti-angiogenic ability of OC. We firstly detected its effect on the motility of HUVECs using the Transwell assay. Results showed that OC significantly decreased the migration and invasion abilities of HUVECs in a dose-dependent manner (Fig. 4A and B). For further insight into the role of $\mathrm{OC}$ on angiogenesis, tube formation assay of HUVECs was performed. We found that OC significantly suppressed tube formation ability of HUVECs in a dose-dependent manner (Fig. 4C and D). The process of angiogenesis also requires the proliferation of endothelial cells, so the effect of OC on proliferation of HUVECs was also detected. Results showed that OC suppressed the cell viability of HUVECs in a dose-dependent manner (Fig. 4E). 

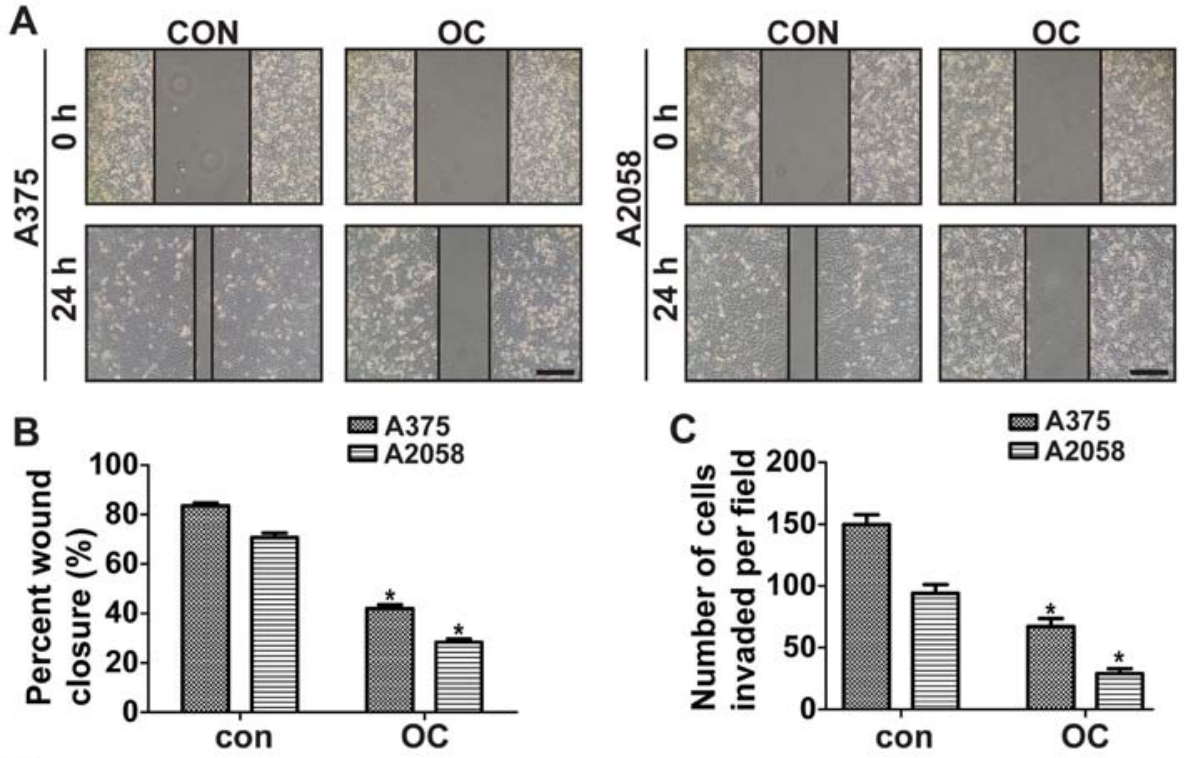

A375
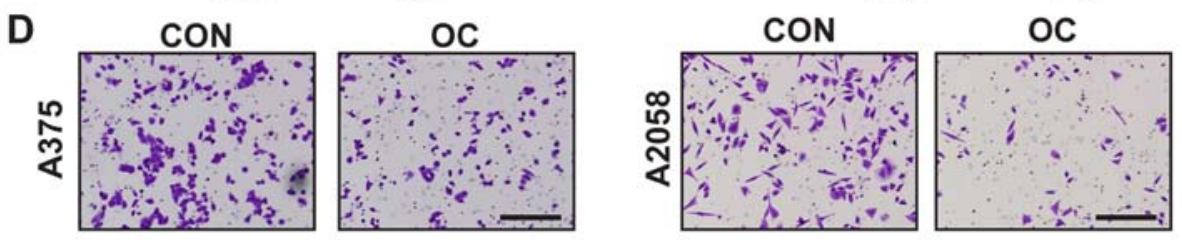

Figure 3. OC inhibits migration and invasion of melanoma cells. (A and B) Single scratch was created in the confluent monolayer of melanoma cells. The scratch was photographed at 0 and $24 \mathrm{~h}$ after treatment with $10 \mu \mathrm{M}$ of OC. Representative photographs are shown and relative migrated areas were analyzed by ImageJ software. Scale bar, $50 \mu \mathrm{m}$. (C and D) Melanoma cells were treated with $10 \mu \mathrm{M}$ of OC, and the invasive capacity was determined by the Transwell invasion assay. Representative photographs of invasive cells and quantification of invasive cells are shown. Scale bar, $100 \mu \mathrm{m}$. Data are presented as the means \pm SD of three independent experiments. ${ }^{*} \mathrm{P}<0.05$.

OC inhibits constitutive STAT3 phosphorylation, and downregulates the expressions of apoptosis-, invasionand angiogenesis-related proteins in melanoma cells. Constitutive activation of STAT3 signaling pathway has been reported to play a key role in the growth, angiogenesis, and metastasis of melanoma (8). Thus, we investigated if OC suppressed the constitutive phosphorylation of STAT3. OC treatment inhibited the phosphorylation of STAT3 at the tyrosine 705 (Tyr705) site in a dose-dependent manner in A375 and A2058 cells, with little impact on the expression of total STAT3 (Fig. 5A). STAT3 is recognized to be activated by upstream tyrosine kinases such as JAK2 and Src in melanoma (32). Therefore, we explored if OC suppressed the activation of JAK2 and Src. OC inhibited the constitutive phosphorylation of JAK2 and Src in A375 and A2058 cells (Fig. 5A). We next investigated whether OC could suppress the nuclear localization of STAT3. After OC treatment, the cytoplasmic and nuclear proteins of melanoma cells were extracted and expression of STAT3 in both fractions were determined by western blotting. The levels of STAT3 in nuclear fractions were decreased in a dose-dependent manner after OC treatment, while those in cytoplasmic fractions were slightly increased (Fig. 5B). To detect whether OC affected STAT3-mediated transcriptional activity, a STAT3-luciferase reporter construct harboring four copies of STAT3 binding sites was transfected into melanoma cells. Then, melanoma cells were treated with indicated concentrations of OC for $24 \mathrm{~h}$ before testing the transcriptional activity by luciferase assay. OC inhibited the STAT3-luciferase reporter activity in a dose-dependent manner (Fig. 5D). Furthermore, OC also downregulated the STAT3-regulated gene products, including anti-apoptotic proteins (Bcl-xL and Mcl-1), invasion-related proteins (MMP-2 and MMP-9), and angiogenesis protein (VEGF) (Fig. 5C).

OC inhibits tumor growth and metastasis of melanoma in vivo. We established a subcutaneous xenograft model in nude mice using A375 cells to determine the effects of OC on melanoma growth in vivo. Compared with the control group, $\mathrm{OC}$ treatment resulted in a significant decrease of tumor size (Fig. 6A). We further investigated the effect of $\mathrm{OC}$ on the expression of Ki-67 (a marker of proliferation) and CD31 (a marker of angiogenesis) in melanoma tumor tissues by immunohistochemical analysis and found that OC significantly decreased the number of Ki-67-positive tumor cells and the microvessel density, compared with the control group (Fig. 6B and C). Furthermore, we investigated expression of tumor-related proteins in nude mice by using western blotting, and the results were consistent with in vitro data (Fig. 6D). The effect of OC on the metastatic ability of melanoma was explored in vivo by injecting luciferase-expressing A375 cells into the tail vein of nude mice to establish a lung metastasis model. We detected stronger illumination signals in control group than that in the OC-treated group (Fig. 6E). At the end of treatment, lungs were excised to perform hematoxylin and eosin staining, and lung micrometastases were microscopically evaluated. Fewer and smaller metastatic foci were detected in the OC-treated group, compared with the control group (Fig. 6F). 
A

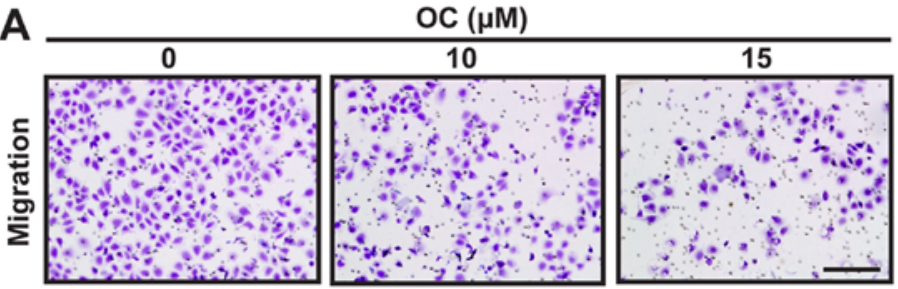

B

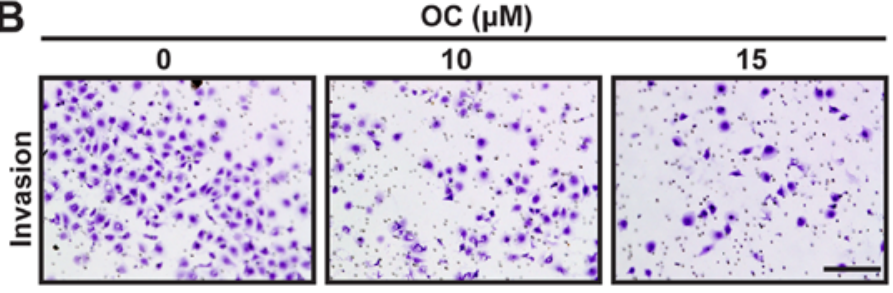

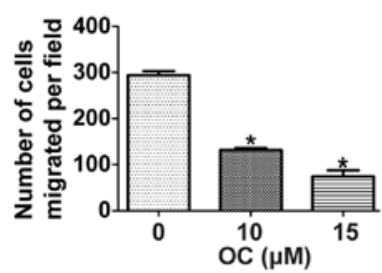

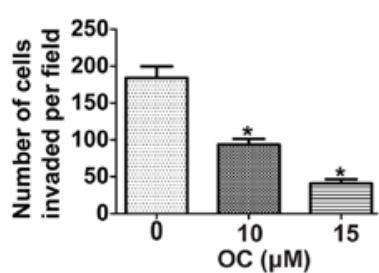

\section{C}
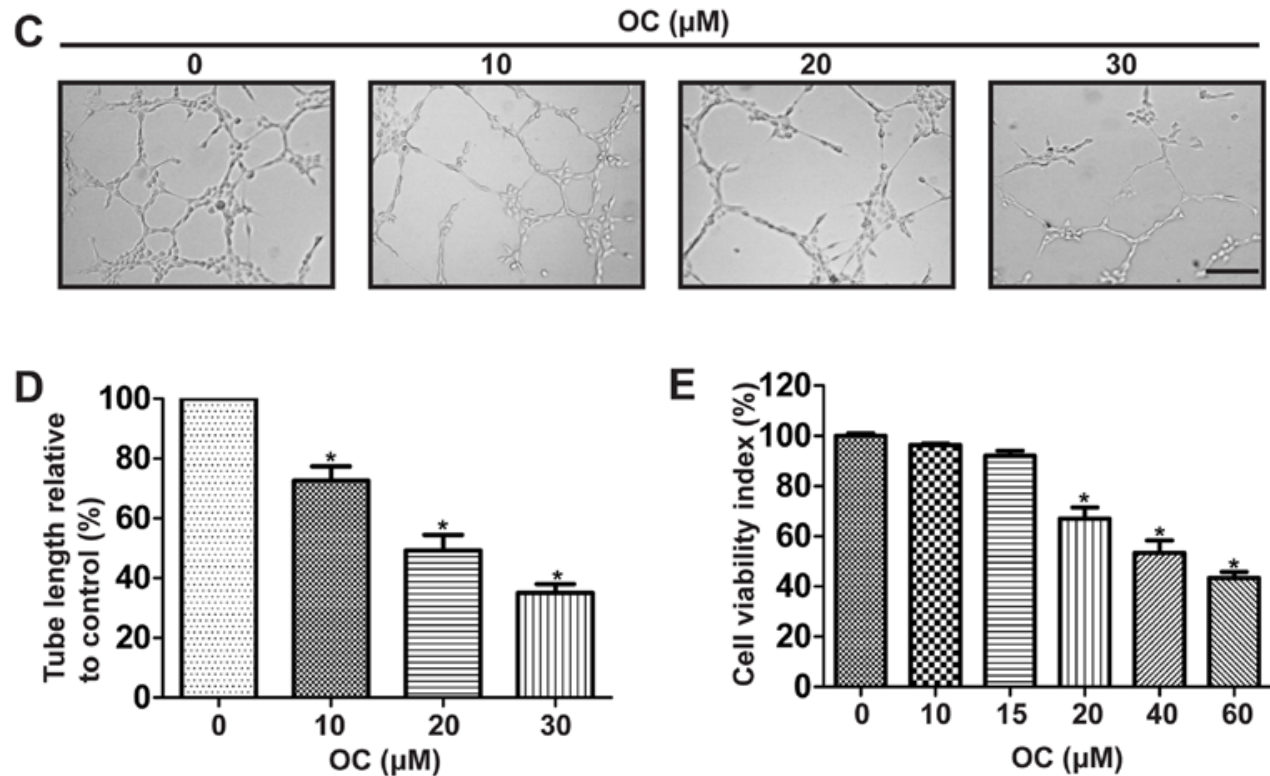

Figure 4. OC suppresses proliferation, migration, invasion and tube formation of HUVECs. (A) Representative images of migration assay for the HUVEC cells after the treatment with increasing doses of $\mathrm{OC}$ for $24 \mathrm{~h}$ (left panel). The number of migrated cells was counted (right panel). Scale bars, $100 \mu \mathrm{m}$. (B) Representative images of invasion assay for the HUVECs after the treatment with increasing doses of OC for $24 \mathrm{~h}$ (left panel). The number of invaded cells was counted (right panel). Scale bars, $100 \mu \mathrm{m}$. (C) Tube formation assay was performed for angiogenesis under various concentrations of OC in HUVECs. Scale bars, $100 \mu \mathrm{m}$. (D) Total tube length per well was calculated in micrometers and changes displayed graphically as a percentage of control. (E) Cell viability was determined by CCK8 assay after the treatment of OC for $24 \mathrm{~h}$. Data are expressed as the mean \pm SD of three independent experiments. ${ }^{*} \mathrm{P}<0.05$.

\section{Discussion}

$\mathrm{OC}$ is a main phenolic compound contained in VOO, and has a long history of consumption as part of the Mediterranean diet. Many biological and pharmacological effects that may be beneficial to human health have been attributed to OC, including antitumor activity $(21,23,24)$. However, the role of $\mathrm{OC}$ in melanoma and the potential molecular mechanisms are not clear. In the present study, we found that OC suppressed proliferation, migration, invasion, angiogenesis and induced apoptosis in melanoma in vitro and in vivo. The underlying mechanisms may be, at least in part, due to the inhibition of STAT3 activation and downregulation of its gene products.

OC are thought to exhibit anti-proliferation effects on cancer cells due to its ability to induce cell cycle arrest and apoptosis. Akl et al reported that OC arrests breast cancer cells at G1/M phase by modulating the expression of CDK6, cyclin D1, p21 and p27 (25). Moreover, OC induces cell apoptosis by activating caspase pathway in MDA-MB-231 cells (25). Based on our study, OC suppressed proliferation and promoted apoptosis in melanoma cells in vitro, but showed no apparent effect on the cell cycle. Furthermore, the slight effect of OC on HaCaT cells in the treatment suggests that it is a compound that specifically suppresses the proliferation of melanoma with negligible side effects. In addition, OC treatment increased the expression of cleaved-caspase-3, cleaved-caspase-9 and cleaved-PARP, which are critical factors in the caspase pathway. More importantly, a pancaspase inhibitor (Z-VAD-FMK) can reverse the effects of $\mathrm{OC}$ on apoptosis and the expression of cleaved-caspase- 3 in melanoma cells. These data showed that OC induces apoptosis in melanoma cells, partially in a caspase-dependent manner.

Angiogenesis, the formation of nascent blood vessels from pre-existing vasculature, is vital for tumor growth and metastasis 
A

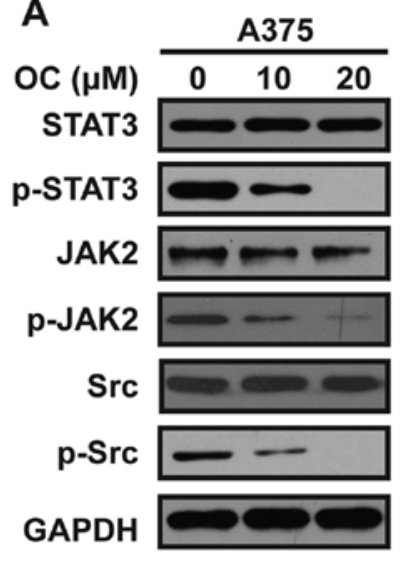

B
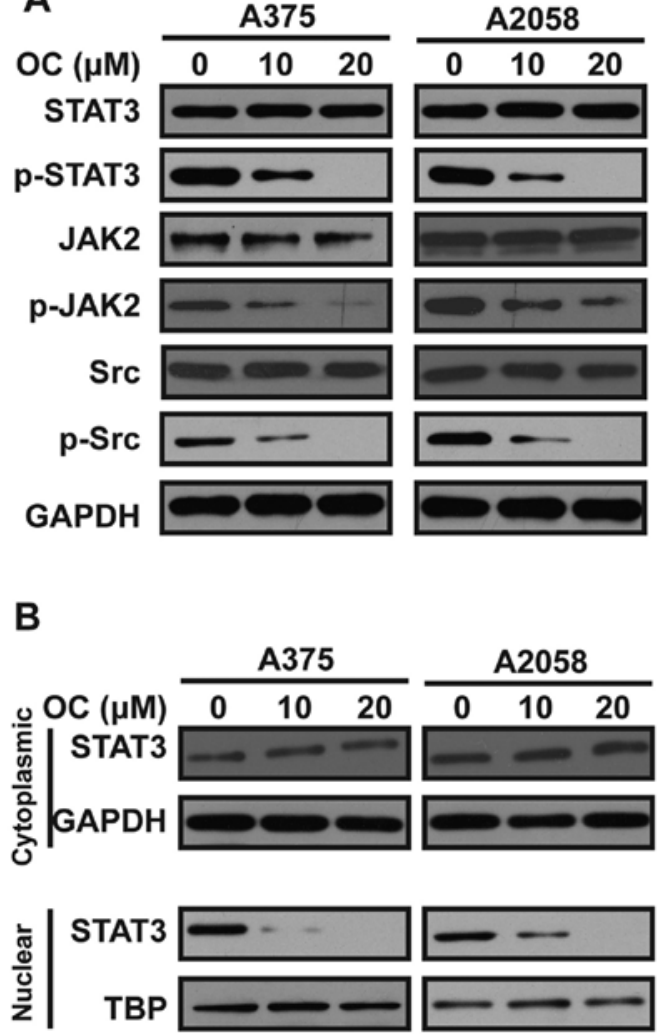

C $O C ( \mu \mathrm { M } ) \longdiv { 0 \quad 1 0 \quad 2 0 }$

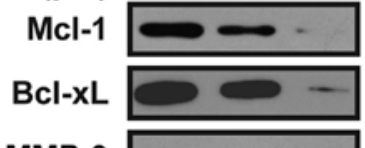

MMP-9

MMP-2

VEGF

GAPDH
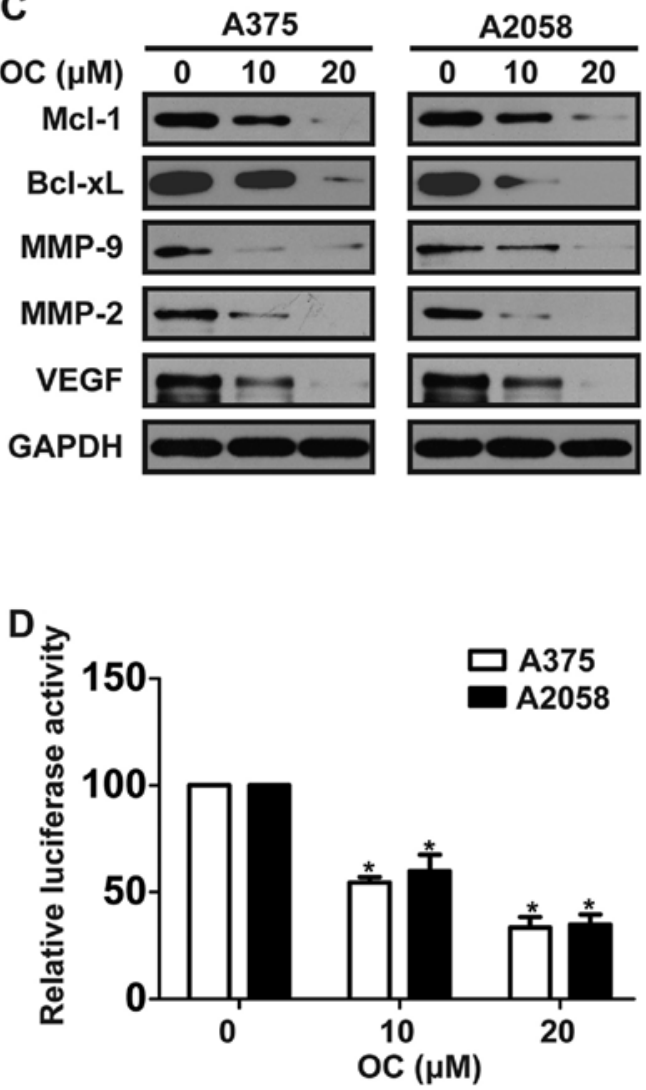

Figure 5. OC inhibits constitutive STAT3 phosphorylation, and downregulates the expressions of apoptosis-, invasion- and angiogenesis-related proteins in melanoma cells. (A) Melanoma cells were treated with various concentrations of OC for $48 \mathrm{~h}$, and then total cell lysates were extracted for western blotting by using antibodies specific to p-STAT3 (Tyr705), STAT3, p-Src (Tyr416), Src, p-JAK2 (Y1007/1008) and JAK2. (B) Melanoma cells were treated with various concentrations of OC for $48 \mathrm{~h}$, expression levels of STAT3 in cytoplasmic and nuclear extracts were examined by western blotting. (C) Melanoma cells were treated with various concentrations of OC for $48 \mathrm{~h}$, and then total cell lysates were extracted for western blotting by using antibodies specific to Mcl-1, Bcl-xL, MMP-9, MMP-2 and VEGF. (D) Melanoma cells were cotransfected with STAT3-Luc reporter and Renilla luciferase reporter plasmids for 48 h, and then cells were treated with indicated concentrations of $\mathrm{OC}$ for $24 \mathrm{~h}$ and the luciferase intensity was measured. Data are expressed as the mean $\pm \mathrm{SD}$ of three independent experiments. ${ }^{*} \mathrm{P}<0.05$.

of malignant tumors (31). This process supplies adequate oxygen and nutrition to support the growth of tumor mass, and later aid in the initiation of metastasis, which contributes to $>90 \%$ of deaths in various cancers, including melanoma. Angiogenesis is initiated by endothelial cell proliferation, migration, invasion and then tube formation (33). It has been shown that inhibition at any step of the processes in angiogenesis will suppress the formation of new blood vessels (34). In the present study, we found that OC significantly suppressed proliferation, migration, invasion and tube formation of HUVECs in vitro, and downregulated the expression of CD31 in the subcutaneous xenograft model. These data suggest that OC may be a promising anti-angiogenic drug with significant antitumor activity in melanoma.

As a previous study reported that OC inhibited the growth and metastasis of HCC through the block of STAT3 signaling pathway (28), we detected the effect of OC on the activation of STAT3. Our data showed that OC significantly decreased the expression of p-STAT3, STAT3 nuclear translocation and STAT3 transcriptional activity in melanoma cells. STAT3 is reported to be activated by upstream tyrosine kinases such as JAK2 and Src in melanoma (32), thus we further explored the expression of JAK2, Src and their phosphorylated forms after OC treatment. OC was found be able to reduce the expression of p-JAK2 and p-Src in a dose-dependent manner in melanoma cells, with little impact on the expression of total JAK2 and Src. In addition, OC also reduced the expression of the STAT3regulated gene products, which are associated with apoptosis, invasion and angiogenesis.

The anti-apoptotic genes Mcl-1 and Bcl-xL are reported to be upregulated in the progression of melanoma (35). In patient samples, the expressions of $\mathrm{Bcl}-\mathrm{xL}$ and $\mathrm{Mcl}-1$ were positively corrected with p-STAT3 expression (36). Suppression of STAT3 can result in the decrease of Mcl-1 and Bcl-xL (37). In this study, we demonstrated that OC suppressed the expressions of Mcl-1 and Bcl-xL in a dose-dependent manner. This suggested that other than activating the caspase pathway, OC-induced apoptosis in melanoma cells was at least partly by inhibiting the expression of Bcl-xL and Mcl-1.

VEGF is a key pro-angiogenic factor that plays a key role in tumor angiogenesis. High serum VEGF values are associated with shorter disease-free survival of melanoma patient (38). STAT3 has been reported to directly participate in regulating transcription of VEGF gene (11). According to our results, OC treatment reduced the expression of VEGF in melanoma cells, suggesting that the anti-angiogenesis effect of OC on melanoma is associated with VEGF inhibition.

MMP-2 and MMP-9 are invasion-related proteins by digesting the extracellular matrix surrounding the tumor 
A

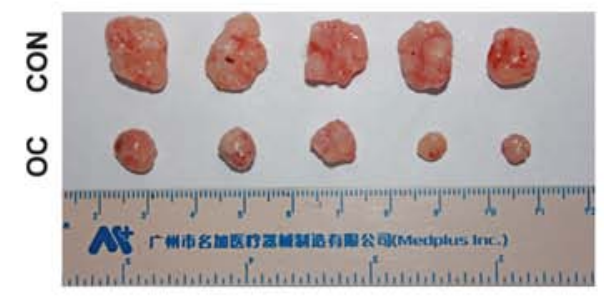

B
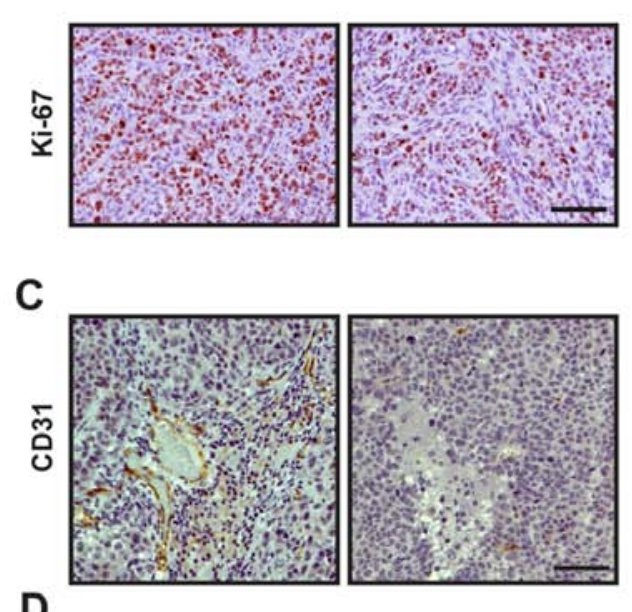

D

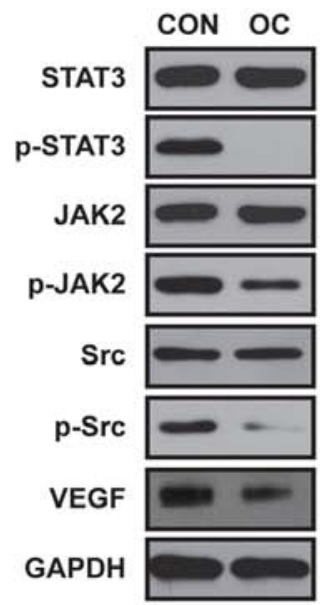

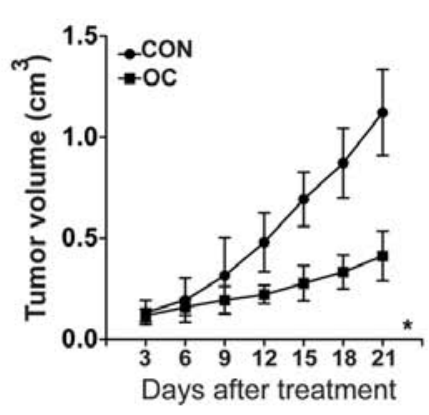

E
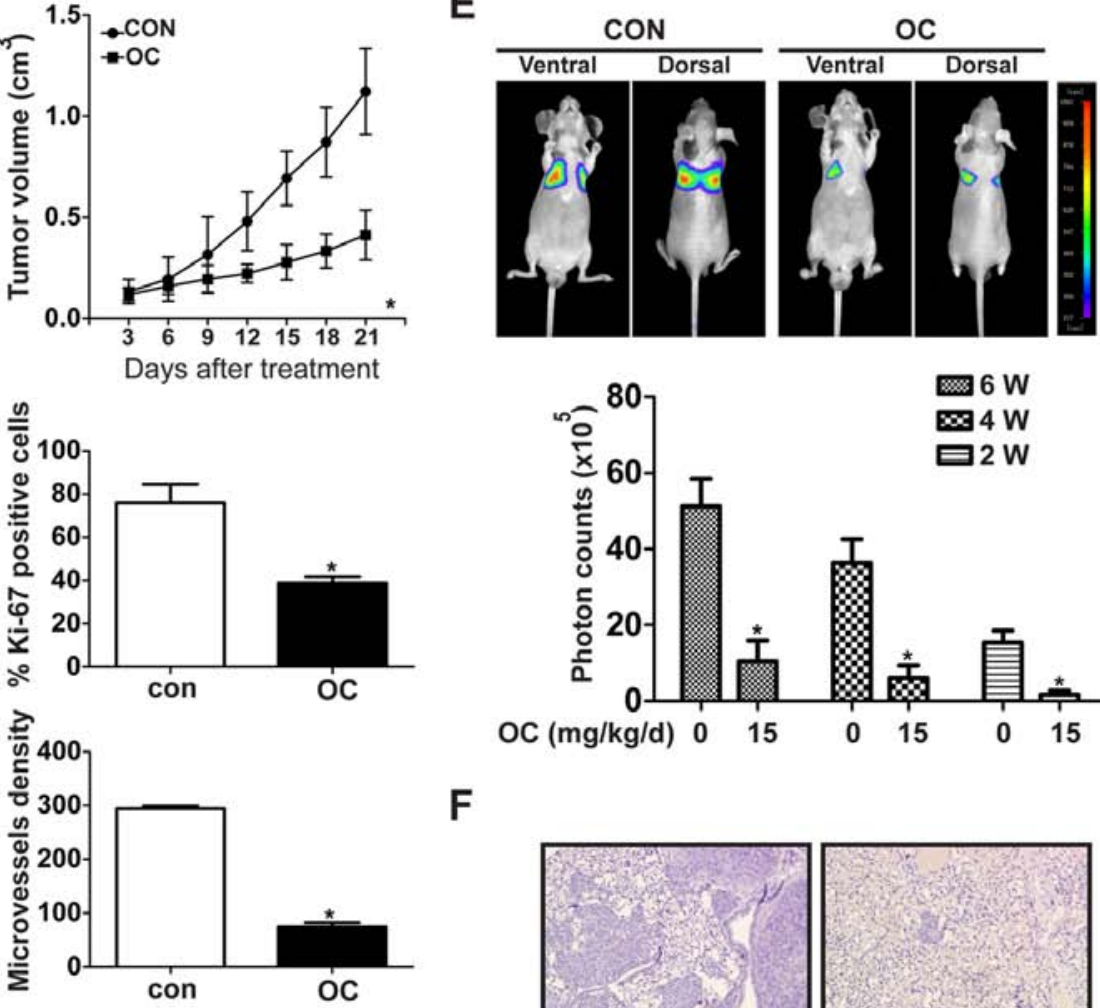

$\mathbf{F}$

CON OC

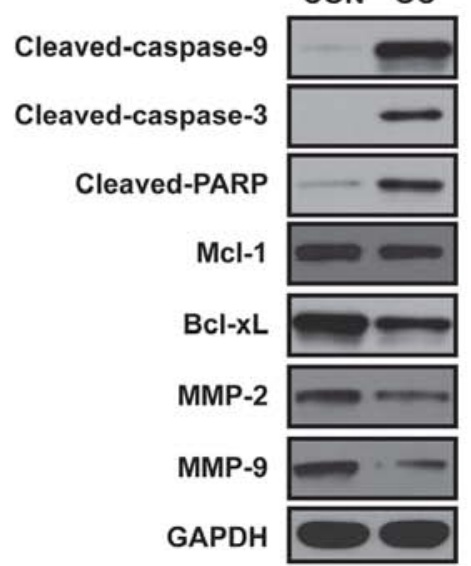

OC $(\mathrm{mg} / \mathrm{kg} / \mathrm{d}) \quad 0 \quad 15$
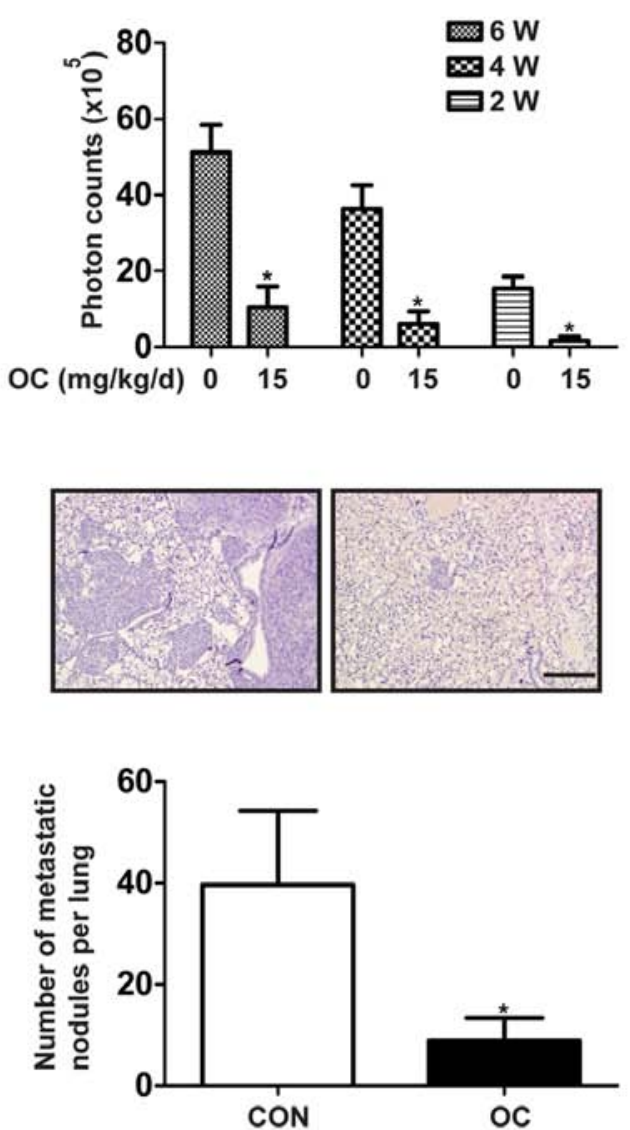

Figure 6. OC inhibits tumor growth and metastasis of melanoma in vivo. (A) Nude mice bearing A375 xenograft tumors were treated with either DMSO or OC $(10 \mathrm{mg} / \mathrm{kg} /$ day) for 3 weeks. Representative tumors removed from mice are shown (left) and tumor volume were measured (right). (B) Immunohistochemical analysis of $\mathrm{Ki}-67$ for cell proliferation in tumor tissues. Ki-67-positive cells were counted to evaluate the proliferation index. Scale bars, $200 \mu \mathrm{m}$. (C) Immunohistochemical analysis of CD31 for angiogenesis in tumor tissues. Microvessels density was counted. Scale bars, $200 \mu \mathrm{m}$. (D) Indicated proteins of tumor samples were detected using western blotting. (E) Luciferase-expressing A375 cells were injected into the nude mice through the tail vein. Animals were randomized to receive either OC $(10 \mathrm{mg} / \mathrm{kg} / \mathrm{day})$ or DMSO at 1 week after injection. The luciferase intensity was detected at the second, fourth and sixth week, respectively. Representative images of mice from bioluminescent imaging at the sixth week are shown in the upper panel, and the luciferase intensity is shown in the bottom panel. (F) Representative hematoxylin and eosin staining are shown in the upper panel, and the number of metastatic foci in the lungs of each group is shown in the bottom panel. Scale bar, $100 \mu \mathrm{m}$. Data are expressed as the mean \pm SD of three independent experiments. ${ }^{*} \mathrm{P}<0.05$.

tissue. Higher expression of MMP-2 is observed in melanoma patients and associated with metastasis of melanoma (39). High serum level of MMP-9 is associated with rapid progression in patients with metastatic melanoma (40). Moreover, it has been reported that inactivation of STAT3 significantly impairs the invasive ability of melanoma cells through decreasing MMP-2 expression (41). Therefore, it is reasonable to deduce that $\mathrm{OC}$ suppressed the migration and invasion of melanoma by reducing the expressions of MMP-2 and MMP9. In line with the deduction, we found that $\mathrm{OC}$ also reduced the expressions of MMP-9 and MMP2. These results indicated that anti-migration and anti-invasion effects of OC on melanoma can be attributed to suppression of MMP-9 and MMP-2.

In conclusion, we showed that OC significantly suppress the constitutive STAT3 activation, which may lead to the suppression of growth and metastasis of melanoma. Although the role of OC on melanoma still needs to be investigated in clinical trials, we propose that $\mathrm{OC}$ may be a potential agent for prevention and therapy of melanoma. 


\section{References}

1. Torre LA, Bray F, Siegel RL, Ferlay J, Lortet-Tieulent J and Jemal A: Global cancer statistics, 2012. CA Cancer J Clin 65: 87-108, 2015

2. Cummins DL, Cummins JM, Pantle H, Silverman MA Leonard AL and Chanmugam A: Cutaneous malignant melanoma. Mayo Clin Proc 81: 500-507, 2006

3. Chi Z, Li S, Sheng X, Si L, Cui C, Han M and Guo J: Clinical presentation, histology, and prognoses of malignant melanoma in ethnic Chinese: A study of 522 consecutive cases. BMC Cancer 11: 85, 2011.

4. Hauschild A, Grob JJ, Demidov LV, Jouary T, Gutzmer R, Millward M, Rutkowski P, Blank CU, Miller WH Jr, Kaempgen E, et al: Dabrafenib in BRAF-mutated metastatic melanoma: A multicentre, open-label, phase 3 randomised controlled trial. Lancet 380: 358-365, 2012.

5. Callahan MK, Postow MA and Wolchok JD: Immunomodulatory therapy for melanoma: Ipilimumab and beyond. Clin Dermatol 31: 191-199, 2013

6. Poust JC, Woolery JE and Green MR: Management of toxicities associated with high-dose interleukin-2 and biochemotherapy. Anticancer Drugs 24: 1-13, 2013.

7. Lopez-Bergami P, Fitchman B and Ronai Z: Understanding signaling cascades in melanoma. Photochem Photobiol 84: 289-306, 2008

8. Kortylewski M, Jove R and Yu H: Targeting STAT3 affects melanoma on multiple fronts. Cancer Metastasis Rev 24: 315-327, 2005.

9. Xie TX, Huang FJ, Aldape KD, Kang SH, Liu M, Gershenwald JE, Xie K, Sawaya R and Huang S: Activation of stat 3 in human melanoma promotes brain metastasis. Cancer Res 66: 3188-3196, 2006.

10. Messina JL, Yu H, Riker AI, Munster PN, Jove RL and Daud AI: Activated stat-3 in melanoma. Cancer control 15: 196-201, 2008

11. Niu G, Wright KL, Huang M, Song L, Haura E, Turkson J, Zhang S, Wang T, Sinibaldi D, Coppola D, et al: Constitutive Stat3 activity up-regulates VEGF expression and tumor angiogenesis. Oncogene 21: 2000-2008, 2002.

12. Liu L, Nam S, Tian Y, Yang F, Wu J, Wang Y, Scuto A, Polychronopoulos $\mathrm{P}$, Magiatis $\mathrm{P}$, Skaltsounis L, et al: 6-Bromoindirubin-3'-oxime inhibits JAK/STAT3 signaling and induces apoptosis of human melanoma cells. Cancer Res 71: 3972-3979, 2011

13. Liu L, Kritsanida M, Magiatis P, Gaboriaud N, Wang Y, Wu J, Buettner R, Yang F, Nam S, Skaltsounis L, et al: A novel 7-bromoindirubin with potent anticancer activity suppresses survival of human melanoma cells associated with inhibition of STAT3 and Akt signaling. Cancer Biol Ther 13: 1255-1261, 2012.

14. Kamran MZ and Gude RP: Pentoxifylline inhibits melanoma tumor growth and angiogenesis by targeting STAT3 signaling pathway. Biomed Pharmacother 67: 399-405, 2013.

15. Martínez ME, Marshall JR and Giovannucci E: Diet and cancer prevention: The roles of observation and experimentation. Nat Rev Cancer 8: 694-703, 2008.

16. Tong LX and Young LC: Nutrition: The future of melanoma prevention? J Am Acad Dermatol 71: 151-160, 2014

17. Fortes C, Forastiere F, Farchi S, Mallone S, Trequattrinni T, Anatra F, Schmid G and Perucci CA: The protective effect of the Mediterranean diet on lung cancer. Nutr Cancer 46: 30-37, 2003

18. Bosetti C, Negri E, Franceschi S, Talamini R, Montella M, Conti E, Lagiou P, Parazzini F and La Vecchia C: Olive oil, seed oils and other added fats in relation to ovarian cancer (Italy). Cancer Causes Control 13: 465-470, 2002.

19. Monti MC, Margarucci L, Tosco A, Riccio R and Casapullo A: New insights on the interaction mechanism between tau protein and oleocanthal, an extra-virgin olive-oil bioactive component. Food Funct 2: 423-428, 2011.

20. Monti MC, Margarucci L, Riccio R and Casapullo A: Modulation of tau protein fibrillization by oleocanthal. J Nat Prod 75: $1584-1588,2012$

21. Lucas L, Russell A and Keast R: Molecular mechanisms of inflammation. Anti-inflammatory benefits of virgin olive oil and the phenolic compound oleocanthal. Curr Pharm Des 17: 754-768, 2011
22. Cicerale S, Breslin PA, Beauchamp GK and Keast RS: Sensory characterization of the irritant properties of oleocanthal, a natural anti-inflammatory agent in extra virgin olive oils. Chem Senses 34: 333-339, 2009

23. Romero C, Medina E, Vargas J, Brenes M and De Castro A In vitro activity of olive oil polyphenols against Helicobacter pylori. J Agric Food Chem 55: 680-686, 2007.

24. Busnena BA, Foudah AI, Melancon T and El Sayed KA: Olive secoiridoids and semisynthetic bioisostere analogues for the control of metastatic breast cancer. Bioorg Med Chem 21: 2117-2127, 2013

25. Akl MR, Ayoub NM, Mohyeldin MM, Busnena BA, Foudah AI, Liu YY and Sayed KA: Olive phenolics as c-Met inhibitors: (-)-Oleocanthal attenuates cell proliferation, invasiveness, and tumor growth in breast cancer models. PLoS One 9: e97622, 2014.

26. Elnagar AY, Sylvester PW and El Sayed KA: (-)-Oleocanthal as a c-Met inhibitor for the control of metastatic breast and prostate cancers. Planta Med 77: 1013-1019, 2011.

27. Scotece M, Gómez R, Conde J, Lopez V, Gómez-Reino JJ, Lago F, Smith AB III and Gualillo O: Oleocanthal inhibits proliferation and MIP-1 $\alpha$ expression in human multiple myeloma cells. Curr Med Chem 20: 2467-2475, 2013

28. Pei T, Meng Q, Han J, Li HS, Song R, Sun B, Pan S, Liang D and Liu L: (-)-Oleocanthal inhibits growth and metastasis by blocking activation of STAT3 in human hepatocellular carcinoma. Oncotarget: Jun 2, 2016 (Epub ahead of print).

29. Smith AB III, Han Q, Breslin PA and Beauchamp GK: Synthesis and assignment of absolute configuration of (-)-oleocanthal: A potent, naturally occurring non-steroidal anti-inflammatory and anti-oxidant agent derived from extra virgin olive oils. Org Lett 7: 5075-5078, 2005.

30. Song R, Song H, Liang Y, Yin D, Zhang H, Zheng T, Wang J, Lu Z, Song X, Pei T, et al: Reciprocal activation between ATPase inhibitory factor 1 and NF- $\mathrm{NB}$ drives hepatocellular carcinoma angiogenesis and metastasis. Hepatology 60: 1659-1673, 2014.

31. Ferrara N and Kerbel RS: Angiogenesis as a therapeutic target. Nature 438: 967-974, 2005.

32. Al Zaid Siddiquee K and Turkson J: STAT3 as a target for inducing apoptosis in solid and hematological tumors. Cell Res 18: 254-267, 2008

33. Fan TP, Yeh JC, Leung KW, Yue PY and Wong RN: Angiogenesis: From plants to blood vessels. Trends Pharmacol Sci 27: 297-309, 2006.

34. Tournaire R, Simon MP, le Noble F, Eichmann A, England P and Pouysségur J: A short synthetic peptide inhibits signal transduction, migration and angiogenesis mediated by Tie 2 receptor. EMBO Rep 5: 262-267, 2004

35. Hartman ML and Czyz M: Anti-apoptotic proteins on guard of melanoma cell survival. Cancer Lett 331: 24-34, 2013.

36. Zhuang L, Lee CS, Scolyer RA, McCarthy SW, Zhang XD, Thompson JF and Hersey P: Mcl-1, Bcl-XL and Stat 3 expression are associated with progression of melanoma whereas Bcl-2, AP-2 and MITF levels decrease during progression of melanoma. Mod Pathol 20: 416-426, 2007

37. Niu G, Bowman T, Huang M, Shivers S, Reintgen D, Daud A, Chang A, Kraker A, Jove R and Yu H: Roles of activated Src and Stat3 signaling in melanoma tumor cell growth. Oncogene 21: 7001-7010, 2002.

38. Ascierto PA, Leonardi E, Ottaiano A, Napolitano M, Scala S and Castello G: Prognostic value of serum VEGF in melanoma patients: A pilot study. Anticancer Res 24: 4255-4258, 2004.

39. Rotte A, Martinka M and Li G: MMP2 expression is a prognostic marker for primary melanoma patients. Cell Oncol (Dordr) 35: 207-216, 2012.

40. Nikkola J1, Vihinen P, Vuoristo MS, Kellokumpu-Lehtinen P, Kähäri VM and Pyrhönen S: High serum levels of matrix metalloproteinase-9 and matrix metalloproteinase-1 are associated with rapid progression in patients with metastatic melanoma. Clin Cancer Res 11: 5158-5166, 2005.

41. Xie TX, Wei D, Liu M, Gao AC, Ali-Osman F, Sawaya R and Huang S: Stat 3 activation regulates the expression of matrix metalloproteinase-2 and tumor invasion and metastasis. Oncogene 23 3550-3560, 2004. 